\title{
Craniectomía descompresiva para empiema por Aggregatibacter aphrophilus: revisión de la literatura y presentación de caso
}

\section{Decompressive Craniectomy for Empyema by Aggregatibacter aphrophilus: Literature Review and Case Report}

Recepción: 25 Enero 2018 | Aceptación: 23 Abril 2018

\author{
Óscar Feo LeE \\ Pontificia Universidad Javeriana, Colombia \\ Sergio I. Patiño-Ladino ${ }^{b}$ \\ Pontificia Universidad Javeriana, Colombia
}

a Medico neurocirujano, Unidad de Neurocirugía, Hospital Universitario San Ignacio. Profesor del Departamento de Neurociencias, Pontificia Universidad Javeriana, Bogotá, Colombia.

b Estudiante de Medicina, Pontificia Universidad Javeriana, Bogotá, Colombia.

\section{RESUMEN}

El empiema subdural es una patología rara que ocurre secundaria a otra infección en el complejo craneofacial; puede ser por una otitis media o por sinusitis en la mayoría de los casos. Por esto, normalmente, los gérmenes asociados con esta patología son los mismos encontrados en el oído medio y en los senos paranasales. A veces, un microrganismo raro causa el absceso, caso de este paciente, quien ingresó al hospital con dolor ocular y rápidamente progresó a un déficit neurológico causado por la bacteria Aggregatibacter aphrophilus. El caso tuvo una rápida intervención de todos los servicios involucrados. Neurocirugía drenó el empiema mediante trepano; posteriormente, el paciente requirió ventriculostomía y craniectomía descompresiva. El objetivo de esta revisión de la literatura es determinar qué dice la evidencia acerca del drenaje de empiemas mediante trepano o el uso temprano de craniectomía en este tipo de pacientes.

\section{Palabras clave}

craniectomía; craneotomía; empiema subdural; Aggregatibacter aphrophilus.

\footnotetext{
ABSTRACT

The subdural empyema is a rare pathology that normally occurs secondary to another infection in the skull-facial complex, could be medial otitis or sinusitis in most of the cases, that's why the germs associated with this pathology are normally the same found in the middle ear and paranasal sinuses. Eventually a rare microorganism causes an abscess, this is the case of this patient, who arrived to the hospital with ocular pain and rapidly progress with neurological deficit cause by a bacteria called Aggregatibacter aphrophilus. The case had a fast intervention of all the services involved, neurosurgery made an empyema drainage by burr hole, after that the patient required ventriculostomy and finally decompressive craniectomy. The objective of this review of the literature is to determine what does the evidence say about the empyema drainage by burr hole or the early use of craniectomy in this kind of patients.

Keywords

craniectomy; craniotomy; subdural empyema; Aggregatibacter aphrophilus.
}

Cómo citar: Feo Lee O, Patiño-Ladino SI. Craniectomía descompresiva para empiema por Aggregatibacter aphrophilus: revisión de la literatura yresentación de caso. Univ. Med. 2019;60(1 cran 


\section{Introducción}

Se define el empiema cerebral como una infección piogénica que se da en un espacio anatómico sin alteraciones previas. Si se habla de empiema subdural, se encuentra entre la duramadre y la aracnoides $(1,2,3)$. Corresponde del 13\% al 23\% de las infecciones encefálicas (3) y representa del $10 \%$ al $40 \%$ de las infecciones supurativas que se presentan en esa área $(2,4)$. Corresponde a una urgencia neuroquirúrgica, debido a que se presenta con un deterioro neurológico rápido (5) y una mortalidad hasta del $17 \%(6)$.

Anatómica y clínicamente, podemos dividir los empiemas en dos grandes grupos: los que se presentan en el encéfalo y la formación de empiemas en las meninges que recubren la médula. Estos últimos se consideran raros y, por esto, hay muy pocos casos clínicos publicados, sin series grandes de pacientes (1). Lo importante de esta diferenciación es la identificación de los signos semiológicos que se presentan en cada caso, lo cual adquiere mayor importancia debido a que muchas veces la sintomatología es inespecífica y dificulta el diagnóstico temprano. Cuando el empiema se presenta en el cerebro se caracteriza por: signos de irritación meníngea (principalmente rigidez nucal), focalización sensitiva o motora, alteración del estado de conciencia, convulsiones, fiebre, signos de hipertensión endocraneana, dolor (facial, ocular o cefalea) y episodios eméticos $(1,2,5,7,8)$. En el caso de una presentación espinal, se tendrían síntomas como fiebre, dolor lumbar, parestesias y disfunción vesical (1), aunque esta patología no es el objetivo de esta revisión.

Se ha descrito que la causa de la formación de empiemas cerebrales está dada por dos mecanismos principales: 1) por contigüidad, en el que se presenta una infección próxima a la masa encefálica que disemina hasta el espacio subdural y genera la formación de la colección, y 2) por vía hematógena, en el que alguna infección sistémica presente diseminación que llegue hasta el cerebro (7). Dado lo anterior, las principales causas de empiema son mastoiditis secundaria a otitis media, sinusitis, meningitis e infecciones dentarias; también se ha asociado con trauma, por la posibilidad de fractura de la pared posterior del seno frontal principalmente, que genera una comunicación directa entre el seno paranasal y la masa encefálica, lo que favorece la aparición de empiema $(2,3,6,8,9,10,11)$.

La clave para el pronóstico y desenlace de los pacientes que cursan con empiema se ha relacionado con un temprano diagnóstico que se siga de un manejo médico-quirúrgico inmediato $(1,4)$. Algunos estudios estiman que desde la instauración del tratamiento temprano de manera agresiva se ha disminuido la mortalidad del $40 \%$ al $10 \%$ (8). La dificultad radica en lo poco específicos que son los síntomas y la cercanía semiológica con otras patologías como la meningitis (4), así como en la gran posibilidad de obtener resultados paraclínicos, como la muestra de líquido cefalorraquídeo sin ninguna alteración $(1,10)$. Por esto, las imágenes diagnósticas constituyen el principal eje diagnóstico de la patología. Se recomienda la toma de una tomografía computarizada (TAC), por su facilidad y rapidez; adicionalmente, porque se considera suficiente para el diagnóstico según algunas series de casos, teniendo en cuenta que pueden obviarse empiemas intracraneales por el artefacto que genera la tabla ósea. Dado lo anterior, el patrón de referencia (gold standard) para el diagnóstico del empiema es la resonancia magnética, específicamente con gadolinio como contraste $(1,2,7,8,10)$.

Posterior a la consecución del diagnóstico, se pasa inmediatamente al tratamiento, que se ha fundamentado en tres ejes principales: 1) manejo antibiótico, el cual varía dependiendo del autor; pero todos coinciden en la necesidad del uso de antibióticos de amplio espectro que cubran los principales microrganismos típicamente causantes de la patología. 2) Manejo quirúrgico que cuenta con un doble propósito: el de descomprimir el edema e inflamación que se está produciendo en la masa encefálica y el de obtener muestras directas de la colección purulenta, con el objetivo de tipificar el germen causal y ajustar el manejo antibiótico. 3) Drenaje del empiema, con el objetivo de frenar la diseminación de la infección, disminuir la 
inflamación y disminuir al máximo la posibilidad de reaparición. (1,2,10). La evaluación del manejo quirúrgico corresponde a determinar la efectividad de las principales técnicas usadas actualmente: la realización de un trepano por el que se drena el pus y se irriga el empiema y el uso de una craniectomía o craneotomía, a fin de liberar mayor espacio para el edema del encéfalo $(2,9,12)$.

\section{Reporte de caso}

El caso corresponde a un joven de 15 años de edad que ingresó al Hospital Universitario San Ignacio con un cuadro clínico de dos semanas de evolución de dolor en el ojo izquierdo asociado a epífora, con dificultad para la apertura ocular y secreción palpebral sin afectación de los movimientos oculares. Adicionalmente, había tenido picos febriles cuantificados hasta en $38^{\circ} \mathrm{C}$, émesis y cefalea pulsátil de inicio reciente que iniciaba periorbitaria y se volvía holocraneana. El paciente manifestó que, previo al inicio del cuadro, había tenido rinorrea y congestión nasal. Había recibido un manejo inicial extrainstitucional con loratadina, diclofenaco y colirio carboximetilcelulosa. No se notificaron antecedentes de importancia ni hubo información relevante en la revisión por sistemas, relacionada con presencia de patología infecciosa otológica, bucal o de senos paranasales reciente.

En la valoración inicial se encontraba taquicárdico, álgico, con edema palpebral leve izquierdo, sin signos meníngeos o alteraciones neurológicas, ni hallazgos sugestivos de otitis o faringitis. Por ese motivo, se dio un diagnóstico inicial de síndrome febril por una posible celulitis postseptal izquierda y se solicitaron, por un lado, valoración por parte del servicio de oftalmología; por otro, exámenes paraclínicos para el estudio de patología infecciosa (tabla 1).

\section{Tabla 1}

Hemogramas y PCR durante la hospitalización

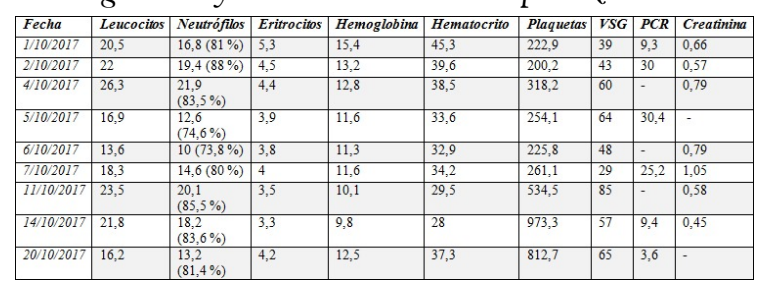

Unidades: leucocitos, neutrófilos y plaquetas $x$

$10^{3}$; eritrocitos $\times 10^{6} ; \mathrm{Hb}: \mathrm{g} / \mathrm{dL}$; hematocrito: $\%$.

El servicio de oftalmología encontró al paciente sin alteración de la agudeza visual, con pupilas simétricas normorreactivas, por lo que se descartó alguna patología oftalmológica; pero por la persistencia de cefalea y aumento en su intensidad fue valorado por el servicio de neurología, que lo detectó con alteración del estado de conciencia y alteración del lenguaje. Por esta razón, en contexto de foco parameníngeo, se solicitó una punción lumbar para estudio del líquido cefalorraquídeo, que se reportó como ligeramente turbio, de color hemático, con 41,4 mg/dL de glucosa, proteínas menores a $300 \mathrm{mg} / \mathrm{dL}$, leucocitos en $9 \times \mathrm{mm}^{3}$ con un $6 \%$ de neutrófilos, $18 \%$ de linfocitos, $1 \%$ de monocitos y no se informaron gérmenes en el Gram.

Paralelamente, el paciente fue valorado por pediatría, el servicio tratante, que al encontrarlo con ptosis palpebral, solicitó un TAC de órbitas y de cráneo contrastado (figura 1). Según los hallazgos, se inició un manejo antibiótico con 1,5 g de ampicilina/sulbactam cada 6 h. Posteriormente, se cambió el manejo a vancomicina IV de $500 \mathrm{mg} / 6 \mathrm{~h}, 1 \mathrm{~g}$ de ceftriaxona cada $12 \mathrm{~h}$ y metronidazol IV de $500 \mathrm{mg}$ cada $8 \mathrm{~h}$ por parte del servicio de infectología pediátrica, en sospecha de infección del sistema nervioso central; por ello, adicionalmente, se solicitó la toma de una resonancia magnética de cerebro (figura 2). Para este momento, el paciente ya presentaba un puntaje de 10/15 en la escala de coma de Glasgow (GCS) con hemiparesia derecha y persistencia de alteración del estado de conciencia, que en conjunto con hallazgos imagenológicos determinaron la valoración por neurocirugía. 


\section{Figura 1}

a) TAC de cráneo: sin alteraciones. b) TAC de órbitas: ocupación completa por material con densidad de tejido blando de celdillas etmoidales anteriores izquierdas y del seno frontal del mismo lado. Importante engrosamiento en el revestimiento mucoso del antro maxilar izquierdo y en menor proporción del seno frontal derecho y esfenoidal izquierdo. También se observa engrosamiento del revestimiento mucoso en celdillas etmoidales anteriores derechas. Sugestivo de celulitis periorbitaria izquierda preseptal. Pansinusitis descrita.
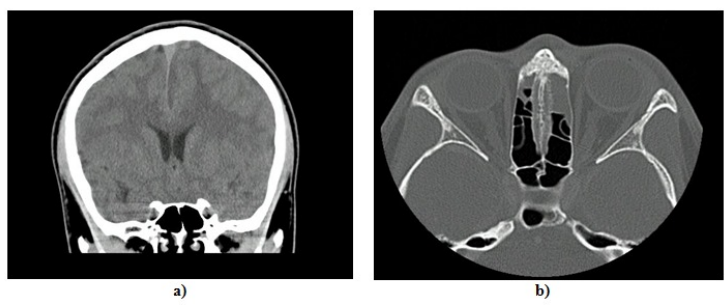

Figura 2

RMN de cerebro con contraste: A nivel de convexidad frontoparietotemporal izquierda con extensión a cisura interhemisférica se aprecia colección subdural. a) Hipointensa en T1.b) Hiperintensa en T2 con fenómeno de restricción a la difusión. c) Realce periférico tras la administración de gadolinio, condicionando desplazamiento de la línea media de $4 \mathrm{~mm}$ en relación con probable empiema subdural asociado a realce paquimeníngeo. Se aprecia realce de surcos de espacio subaracnoideo no descartando HSA, diseminación de colección descrita o infarto a este nivel. d) TAC de cráneo simple: Sin lesiones córtico-subcorticales sugestivos de infartos recientes. Extensa colección subdural supratentorial predominante hipodensa en la convexidad izquierda con extensión a región interhemisférica, generando moderada compresión sobre el parénquima cerebral, disminución en amplitud del ventrículo lateral y desplazamiento de las estructuras de la línea media hacia la derecha de hasta $8 \mathrm{~mm}$. Sin disminución significativa de la amplitud de las cisternas peritroncales. Compatible con infartos córtico-subcorticales en el cíngulo izquierdo en el aspecto anterior de los giros frontales superior y medio del mismo lado y empiema.

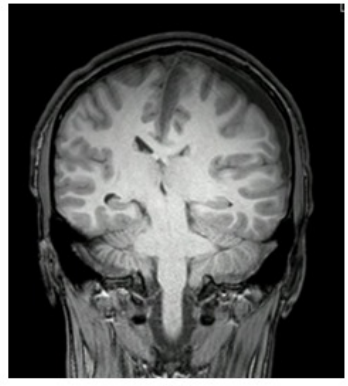

a)

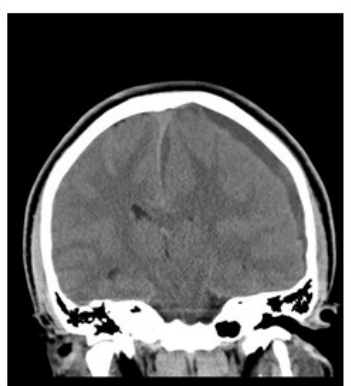

c)

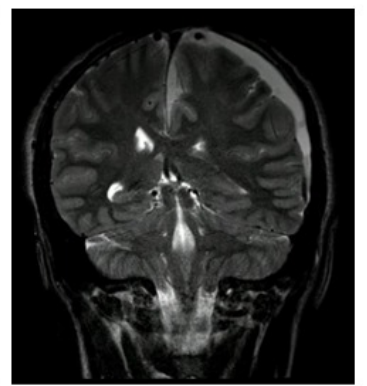

b)

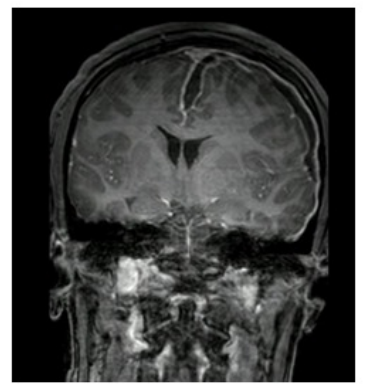

d) 
Fue encontrado bajo sedación farmacológica con un GCS de 3/15, pupilas mióticas hiporreactivas, movimientos oculares con ojos de muñeca, con respuesta plantar neutra bilateral. Con la evaluación de las imágenes tomadas se determinó disfunción hemisférica, por lo que se decide la toma de un nuevo TAC (figura 2d) comparativo y descartar infarto venoso, compromiso ictal o cerebritis asociada a la colección. Se decidió que el paciente requería manejo quirúrgico. Adicionalmente, el servicio de otorrinolaringología decidió manejo quirúrgico para control del foco.

Neurocirugía drenó la expansión subdural por craneotomía haciendo un drenaje controlado del empiema subdural. Se obtuvo líquido cetrino y, posteriormente, pus franca. Se introdujo zonda nelaton y se realizó lavado de la cavidad con 2 L de SSN tibia hasta obtener líquido claro. Posteriormente, otorrinolaringología realizó frontomaxiloidectomía izquierda y encontró secreción purulenta en la nasofaringe. Fue llevado a la unidad de cuidado intensivo pediátrico donde se inició protección cerebral con sedoanalgesia y manejo antiedema con SSN hipertónica 0,5 U/kg/h.

Posterior a la intervención quirúrgica, el paciente tuvo una evolución clínica y neurológica estable, sin cambios en el Glasgow, que se mantuvo en $3 / 15$, con difícil valoración de preservación de reflejos de tallo por sedoanalgesia. En el segundo día del postoperatorio, presentó anisocoria súbita que se resolvió con manejo médico. Fue llevado a toma de TAC en el que se evidenciaron infartos cerebrales múltiples de localización frontal y occipital izquierdos, por lo que se decidió llevarlo nuevamente a cirugía para implantación de catéter ventricular.

Se pasó un catéter ventricular en dos pasos con posterior exteriorización y conexión a un sistema de monitoría y drenaje. Se tomó presión intracraneal que se reportó en $40 \mathrm{~mm} \mathrm{Hg}$ con posterior mejoría del diámetro pupilar (ojo derecho: $2 \mathrm{~mm}$; ojo izquierdo: $3 \mathrm{~mm}$ ). Previo al egreso del quirófano se revaloraron las pupilas, que presentaban una dilatación de $2 \mathrm{~mm}$ en el ojo derecho y de $5 \mathrm{~mm}$ en el ojo izquierdo no reactiva, que no se resolvió a pesar del paso de bolo de manitol, por lo que se consideró una craniectomía descompresiva izquierda (figura 3). Se realizó la apertura y disección del músculo temporal con rechazo del colgajo musculocutáneo hacia inferior. Se realizó craneotomía de $12 \times 11 \mathrm{~cm}$ con fresa y cuchilla de midas rex y ampliación hacia la fosa media con gubia.

\section{Figura 3}

a) Visualización del empiema posterior a durotomía con membranas purulentas, rígidas, gruesas y posterior. b) Lavado

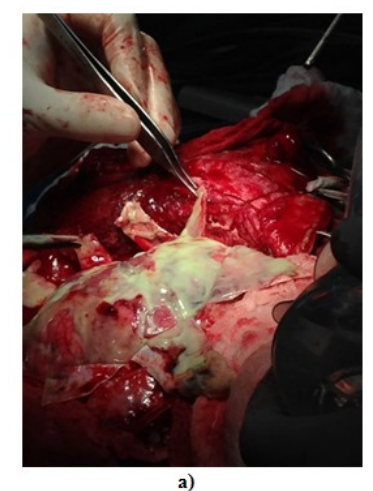

a)

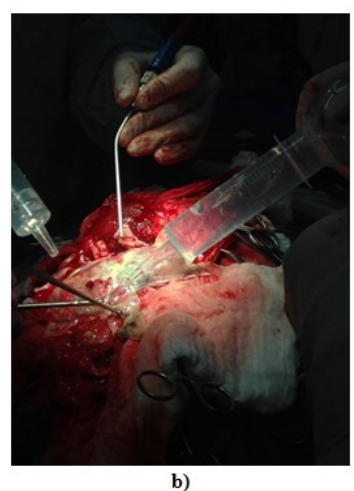

b)
Se realizó durotomía con espina de pescado y se encontró empiema subdural laminar, con múltiples membranas purulentas, rígidas, gruesas y edema cerebral masivo. Se drenó el empiema y las membranas, con posterior lavado con peróxido de hidrógeno y vancomicina diluida en $500 \mathrm{~cm}^{3}$, con evidencia de recuperación de la pulsatilidad cerebral. Posteriormente, se colocó hemostático y se procedió al cierre por planos con lavado profuso de la herida y cubrimiento con gasa estéril y apósito. Se revaloraron las pupilas con mejoría parcial de anisocoria. Posteriormente, fue llevado a la unidad de cuidado intensivo pediátrica.

Posterior al ingreso a la unidad de cuidado intensivo pediátrica, hubo evolución clínica y neurológica estacionaria, con permanente sedoanalgesia $y$, por ende, $3 / 15$ en GCS, pupilas hiporreactivas mióticas. No respondía al estímulo doloroso y el monitoreo de presión intracraneana se encontraba dentro de parámetros normales. Progresivamente, fue 
disminuyendo la leucocitosis, sin nuevos picos febriles.

El servicio de infectología pediátrica decidió tomar una proteína $\mathrm{C}$ reactiva para Staphylococcus aureus e inicio de trimetropina sulfametazolina para dar cubrimiento a este microrganismo como principal sospecha etiológica. Resultó negativo y se suspendió el manejo antibiótico. Al segundo día se recibió reporte de aislamiento de Agregatibacter aphrophilus, que característicamente se presenta en infecciones mixtas, por lo que 6 días después se recibió reporte del microrganismo causante de la coinfección (tabla 2) con Streptococcus intermedius, que se ha asociado a formación de abscesos en el sistema nervioso central; por ese motivo, se inició manejo con metronidazol, ceftriaxona y vancomicina.

Tabla 2

Cronología de reporte de resultados bacteriológicos del empiema

\begin{tabular}{|l|l|l|}
\hline Fecha & Empiema subdural & Antibiograma \\
\hline $3 / 10 / 2017$ & PN ++ & \\
\cline { 2 - 3 } & CGP + & \\
\hline $5 / 10 / 2017$ & Agregatibacter aphrophilus & Multisensible \\
\hline $7 / 10 / 2017$ & & \\
\hline $9 / 10 / 2017$ & & $\begin{array}{l}\text { Sensible a } \\
\text { penicilinas }\end{array}$ \\
\hline $10 / 10 / 2017$ & Streptococcus intermedius & \\
\hline
\end{tabular}

Al tercer día de la cirugía presentó picos febriles hasta de $39{ }^{\circ} \mathrm{C}$ y se reportaron exámenes paraclínicos con aumento de leucocitosis con neutrofilia (tabla 1). Paralelamente, se empezó el descenso de la sedoanalgesia haciendo posible la evaluación de reflejos de tallo, con reflejo carinal y corneano bilateral presente. Por la alteración clínica y paraclínica se decidió la toma de un TAC de control que evidenciaba aumento de la colección interhemisférica (figura 4), que se definió según un concepto de neurocirugía no candidato a manejo quirúrgico. Con el objetivo de definir el foco infeccioso, fue valorado por cirugía maxilofacial en contexto de un microrganismo descrito como flora normal de la cavidad oral. Se le tomó un TAC de cara que presentaba ocupación parcial del seno maxilar izquierdo sin hallazgos que sugirieran procesos infecciosos ontogénicos. El paciente continuó con distermias, por lo que infectología pediátrica amplió estudios con hemocultivos, urocultivos, cultivos de secreción orotraqueal y de línea arterial, así como estudios para determinar inmunodeficiencias de base con reporte de estudios para VIH no reactivos. Adicionalmente, se modificó el esquema antibiótico con la suspensión de ceftriaxona y metronidazol y adición de meropenem 2 g/8h. Se determinó que el tratamiento debía continuar durante 8 semanas.

\section{Figura 4}

TAC postoperatorio. Defecto de craniectomía descompresiva izquierda a través de la cual se aprecia herniación del parénquima encefálico, el cual posee extensas zonas de infartos isquémicos subagudos con trasformación hemorrágica que compromete principalmente la región frontoparietal izquierda y, en menor medida, temporal con edema asociado. Aumento de diámetro de empiema interhemisferico con realce dural saciado, no desplazamiento de línea media, cisternas de la base permeables, catéter de derivación ventricular en asta frontal del ventrículo lateral izquierdo. a) 4 días después de cirugía. b) 7 días después de cirugía.
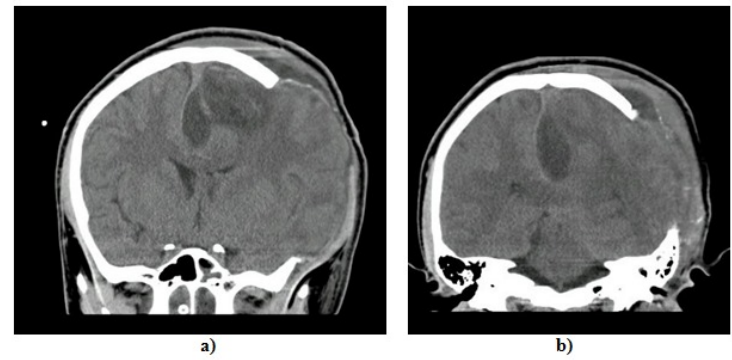

Días después, el paciente continuó con mejoría del estado neurológico con descenso progresivo de sedoanalgesia, con respuesta plantar flexora izquierda y neutral derecha. Posteriormente, recuperó sus movimientos oculares y movimiento de la mano izquierda al estímulo doloroso. Progresivamente, aumentó la movilidad del hemicuerpo izquierdo, obedeciendo órdenes sencillas.

Diez días después de la cirugía, presentó una franca mejoría, con GCS de 11/15, solo con alteración en la respuesta verbal. Tuvo un episodio convulsivo único que fue manejado y resolvió al manejo con levetiracetam, sin 
repercusiones posteriores. Se empezó descenso de fentanil, con el fin de extubarlo y pasarlo a CPAP. Con el objetivo de reinicio de la vía oral, se realizó una videocinodeglución para determinar la permanencia de los reflejos de protección de la vía aérea. Se evidenció broncoaspiración sin reflejo tusígeno, por lo que se consideró la opción de gastrostomía por parte del servicio de gastroenterología pediátrica en conjunto con cirugía pediátrica. El paciente fue trasladado a piso, a la unidad de alta dependencia, a la espera del procedimiento, con estabilidad clínica y neurológica.

\section{Discusión}

Previamente se había discutido la distribución anatómica y clínica de los empiemas que se presentan en el sistema nervioso central. Específicamente, los subtipos que se pueden presentar en el cráneo encontramos el tentorio como límite anatómico para determinar los empiemas supratentoriales y los infratentoriales o de fosa posterior $(2,4,9,10)$. Estos últimos representan del $3 \%$ al $10 \%$ de todos los empiemas $(9,10)$ y de las enfermedades supurativas del cráneo representan solo el 0,6\% (4); por esto, la mayoría de estudios y reportes se han realizado sobre empiemas supratentoriales o hemisféricos (10).

Epidemiológicamente, se encuentran la mayoría de series y reportes de caso en países en vías de desarrollo, sobre todo en India y en Sudáfrica con una importante predominancia en hombres jóvenes $(7,8)$. Además, se han encontrado factores predisponentes y asociados como: VIH, estado postrasplante, tratamiento de enfermedades neoplásicas o enfermedades autoinmunes (12). En el ámbito clínico se ha descrito que una de las principales causas de formación de empiemas subdurales es la presencia de infecciones contiguas que se diseminan y alcanzan el encéfalo. Anatómicamente, por cercanía, se ha descrito que los empiemas subdurales infratentoriales están asociados con infecciones óticas y mastoiditis $(4,7,9)$. Por su parte, los empiemas subdurales supratentoriales están asociados con infecciones en senos paranasales en orden de prevalencia con el seno frontal con la mayor, etmoidal, esfenoidal y maxilar $(5,8,11)$. Semiológicamente, si encontramos compromiso de la fosa posterior, por lo general se presentan signos cerebelosos y de compresión de pares $(2,9)$.

Fisiopatológicamente, se han descrito los mecanismos de diseminación en cada caso. En los empiemas infratentoriales asociados a infecciones óticas se ha descrito una vía directa e indirecta. En el primer caso se produce por erosión del hueso y se ha asociado con empiemas epidurales e infecciones en la tabla ósea. La vía indirecta se da cuando la infección se disemina por los vasos perforantes de la mucosa del oído medio que generan tromboflebitis y producen empiemas subdurales y abscesos cerebelosos (4). Por su parte, en los empiemas supratentoriales hay dos mecanismos de diseminación: está la tromboflebitis retrógrada, que se disemina a través de las venas diploicas del cráneo y del hueso etmoides que tienen más actividad en la juventud, lo que explica la alta prevalencia en hombres jóvenes, o por medio de las venas comunicantes; por otro lado, se puede diseminar por caminos anatómicos anormales secundarios a dehiscencia traumática o malformaciones congénitas (5). Así es como se describe al empiema subdural como una de las principales complicaciones de la sinusitis, adicionándose a meningitis, empiema epidural, absceso intracerebral, tromboflebitis de senos durales, trombosis del seno cavernoso o sagital superior $(5,8)$.

Respecto a la etiología se han descrito múltiples gérmenes causales que se clasifican según el mecanismo de diseminación presentado (tabla 3) asociados en su mayoría a los focos infecciosos y de diseminación mencionados previamente $(5,8)$. La familia del grupo de los Streptococos milleri son característicamente bacterias que constituyen la flora normal de la cavidad oral, y tienen una fuerte asociación con las complicaciones intracraneales de la sinusitis (5) que, como veremos más adelante, corresponde al foco de infección que presentó el paciente descrito en el reporte de caso. 
Tabla 3

Microorganismos causales de empiemas subdurales según foco de diseminación (6)

\begin{tabular}{|l|l|}
\hline Fuente de la infección & Bacteria asociada \\
\hline Senos paranasales & $\begin{array}{l}\text { Estreptococo no hemolítico, Staphylococcus } \\
\text { aureus, Bacteroides fragilis, Staphylococcus } \\
\text { albus, Streptococcus viridans }\end{array}$ \\
\hline Senos mastoideos & $\begin{array}{l}\text { Anaerobios, proteus, estreptococo no hemolítico, } \\
\text { cocos grampositivos, estreptococo betahemolítico }\end{array}$ \\
\hline $\begin{array}{l}\text { Antecedente de trauma } \\
\text { craneal }\end{array}$ & $\begin{array}{l}\text { Escherichia coli, anaerobios, estreptococo no } \\
\text { hemolítico }\end{array}$ \\
\hline Cirugía previa & Estafilococo albus, Streptococcus pneumonie \\
\hline Abscesos previos & Estafilococo albus, anaerobios \\
\hline Diseminación metastásica & Estreptococo no hemolítico, estreptococo milleria \\
\hline Desconocido & $\begin{array}{l}\text { Streptococcus pneumonie, estreptococo } \\
\text { microaerofilico }\end{array}$ \\
\hline
\end{tabular}

El microorganismo causal del empiema subdural que presentó el paciente es el Agregatibacter aphrophilus. Se describe en la literatura como un bacilo gramnegativo cortooxidasa y catalasa negativo $(13,14)$ que hace parte de la flora normal de la cavidad oral, aunque se ha encontrado en otras regiones anatómicas con la producción de colecciones purulentas en peritoneo, pleura y hueso, asociado también a la endocarditis y con menor frecuencia se ha aislado en procesos de meningitis, otitis media, bacteriemia, neumonía y, como en el caso del paciente, en procesos de sinusitis, frecuentemente con coinfección de otro tipo de microorganismo aerobio o anaerobio (13).

Se informa que solo el $2 \%$ de las infecciones cerebrales se asocian con enfermedades dentarias (13), por lo que no es una patología que se presente con frecuencia; por esto, desde 1964 se han registrado 14 reportes de absceso cerebral secundario a infección por esta bacteria (14). De estos, se notificó una mortalidad del 20\% con tres casos, en dos de ellos con patologías cardiacas de base previas al diagnóstico, y en el último con un manejo exclusivo médico con antibióticos. En los 11 reportes adicionales se planteó un manejo dual con cirugía para drenaje de la colección purulenta y antibioticoterapia agresiva (14). Adicionalmente, se ha encontrado asociación con la flora bacteriana bucal normal de los caninos, en quienes es común encontrar esta bacteria, por lo que indagar por el contacto con perros resulta importante al momento de la construcción de la anamnesis (14).

Para el tratamiento de esta patología hay que tener en cuenta los tres ejes mencionados.
Con el abordaje quirúrgico y médico con antibioticoterapia, teniendo en cuenta la división anatómica descrita, se reporta un cambio en la supervivencia, comparando el abordaje quirúrgico con el manejo médico exclusivo del $20 \%$ al $82 \%$ (1). Los estudios y las series de casos, así mismo, se dividen en abordajes para empiemas subdurales supratentoriales, como el presentado por el paciente de este caso, y los infratentoriales.

Como principio quirúrgico se ha descrito que el manejo inicial de todo empiema subtentorial debe ser con craniectomía descompresiva suboccipital $(1,2,9)$, especialmente cuando la colección se extiende hasta el ángulo pontocerebeloso (9), haciendo hincapié en que los empiemas subdurales infratentoriales no son ni deben ser manejados con trepano (12) en el contexto de una patología de rápido crecimiento con efecto de masa importante que genera compresión y repercusión clínica rápida, con repercusiones neurológicas importantes y una alta mortalidad de hasta el $45 \%$ y con una morbilidad del $20 \%$ a pesar del manejo agresivo dual $(3,4,7)$.

Para el abordaje de los empiemas supratentoriales se plantea la discusión y el tema central de la mayoría de los estudios y series de casos: ¿qué resulta más efectivo: la craniectomía o la craneotomía en comparación con el trepano? $(2,6,12)$. La literatura ha comparado ambos abordajes. El estudio de Bannister evidenció una menor mortalidad en los pacientes que fueron llevados a craniectomía en comparación a los que recibieron manejo con trepano $(2,6)$. Madhugiri et al. (12) compararon estas dos intervenciones en 4 de las principales patologías infecciosas supurativas del cráneo: empiemas y abscesos tanto supra como infratentoriales, evaluando el porcentaje de recurrencia de la colección y la infección. Encontraron en todos los casos una menor tasa de recurrencia en la craniectomía (12). Así es como Feurman (2) reportó que el $40 \%$ de los pacientes que recibieron un manejo inicial con trepano requieren una craniectomía posterior por no drenaje completo del empiema, lo cual corresponde a la situación presentada en el caso reportado. 
Por lo anterior, se han propuesto una serie de condiciones específicas en las que se indica el manejo con trepano, siendo la craniectomía temprana el procedimiento más efectivo y que se viene instaurando como tendencia desde los años ochenta (6). Estas son: cuando se presenta un absceso con pared débil y pobremente formada, cuando el GCS es bajo, cuando el paciente presenta un deterioro neurológico rápido y cuando se presenta anemia severa (12). Al evaluar el caso presentado, se decidió el abordaje inicial de un trepano en el contexto de un paciente con GCS bajo y deterioro neurológico rápido. La existencia de bajo volumen de pus, tal es el caso del reporte de caso presentado por Alimehmeti et al. (9), en el que se logró drenaje completo de la colección mediante trepano con recuperación clínica y neurológica.

Cuando se encuentran condiciones como colecciones multiloculadas y volumen de pus importante, se debe considerar craniectomía por la posibilidad de no drenar completamente el absceso que se presente recidiva $(2,4)$. Dentro de la literatura evaluada se encontró un procedimiento alternativo a los dos mencionados, que ofrece una posibilidad terapéutica diferente para los pacientes que cursan con esta patología. Se denomina craneotomía interna y consiste en la descompresión encefálica mediante la liberación de la tabla ósea posterior del seno frontal, con posterior drenaje del material mucoso que allí se encuentre para que el parénquima encefálico pueda ocupar este volumen adicional. Se describe como un procedimiento reciente, al cual le faltan más estudios para instaurarlo como un protocolo de manejo (11).

El desenlace que presentan los pacientes que cursan con esta patología depende de muchas variables: hay que tener en consideración las comorbilidades y condiciones basales prequirúrgicas del paciente (4) , así como el tipo de intervención que se realiza (5). Dentro de los desenlaces postoperatorios que se pueden esperar se encuentran: debilidad de extremidades ipsilaterales o contralaterales a la lesión, hipertensión endocraneana, convulsiones y deterioro cognitivo $(2,12)$. En definitiva, el reporte referido por Venkatesh et al. (4) define los desenlaces que pueden presentar los pacientes que sufren de esta patología con una menor mortalidad en pacientes con adecuado estado de conciencia en el preoperatorio y mayor en quienes se diagnosticó alteración del estado de conciencia y coma.

\section{Conclusión}

El empiema subdural es una entidad rara que constituye una urgencia neuroquirúrgica, por su rápida progresión y diseminación, tanto que llega a afectar severamente el estado neurológico de los pacientes. Es de etiología multifactorial y multicausal, con predominio por las infecciones de contigüidad tanto sinusitis como otomastoiditis para las diferentes localizaciones anatómicas de la colección. A pesar de que claramente están tipificados y estudiados los gérmenes más asociados con esta patología, pueden presentarse casos excepcionales y poco frecuentes como el que se reporta en este documento, por lo que es importante siempre tener en cuenta los microrganismos que pueden llegar a causar un empiema subdural.

El manejo de esta patología está claro, en el sentido de la aplicación que se dé de los tres ejes, enfocándose en el manejo farmacológico con antibióticos de amplio espectro en principio y cubrimiento adecuado posterior a la tipificación del microrganismo causal en conjunto con el manejo quirúrgico. Este último representa un punto de discusión en el abordaje, debido a que mientras en empiemas subdurales infratentoriales está claro el abordaje con craniectomía, en los supratentoriales no se ha establecido un abordaje temprano definitivo entre la realización de un trepano y una craniectomía o craneotomía, a pesar de que la literatura avala y refuerza la realización de este último.

\section{Referencias}

1. Pompucci A, Bonis P de, Sabatino G, Federico G, Moschini M, Anile C, 
et al. Cranio-spinal subdural empyema due to $S$. intermedius: A case report. J Neuroimaging. 2007; 17(4):358-60.

2. Salunke PS, Malik V, Kovai P, Mukherjee KK. Falcotentorial subdural empyema: Analysis of 10 cases. Acta Neurochir (Wien). 2011; 153(1):164-9.

3. Polyzoidis KS, Vranos G, Exarchakos G, Argyropoulou MI, Korantzopoulos P, Skevas A. Subdural empyema and cerebellar abscess due to chronic otitis media. Int J Clin Pract. 2004; 58(2):214-7.

4. Venkatesh MS, Pandey P, Devi BI, Khanapure K, Satish S, Sampath S, et al. Pediatric infratentorial subdural empyema: analysis of 14 cases. J Neurosurg. 2006; 105(5 Suppl):370-7.

5. Kombogiorgas D, Seth R, Athwal R, Modha J, Singh J. Suppurative intracranial complications of sinusitis in adolescence: Single institute experience and review of literature. $\mathrm{Br}$ J Neurosurg. 2007; 21:603-9.

6. Miller ES, Dias PS, Uttley D. Management of subdural empyema: a series of 24 cases. J Neurol Neurosurg Psychiatr [Internet]. 1987; 50(11):1415-8. Disponible en: http://w ww.pubmedcentral.nih.gov/articlerend er.fcgi? artid $=1032550$ \& tool $=$ pmcent rez\&rendertype $=$ abstract

7. Taha MM, Hassanain S. Subtentorial subdural empyema: report of two cases and review of the literatures. J Turkish Neurosurg. 2010; 21(4):669-73.

8. Ong YK, Goh KYC, Chan C. Bifrontal decompressive craniectomy for acute subdural empyema. Child's Nerv Syst. 2002; 18(6-7):340-3.

9. Alimehmeti R, Seferi A, Stroni G, Sallavaci S, Rroji A, Pilika K, et al. Burr hole evacuation for infratentorial subdural empyema. Worl J Clin Cases.
2013; 1(5):172-6. doi: https://doi.org/1 0.12998/wjcc.v1.i5.172.

10. Borovich B, Johnston E, Spagnuolo E. Infratentorial subdural empyema: clinical and computerized tomography findings. J Neurosurg. 1990; 72(2):299-301.

11. Visocchi M, Esposito G, Maria G, Pepa D, Doglietto F, Ginevra C, et al. Giant frontal mucocele complicated by subdural empyema treatment of a rare association. Acta Neurol Belg. 2012; 112:85-90.

12. Madhugiri VS, Savitr B V, Srikantha U. Focal intradural brain infections in children: an analysis of management. Pediatr Neurosurg. 2011; 560029:113-24.

13. Bogdan M, Zujić Atalić VZ, Hećimović I, Vuković D. Brain abscess due to Aggregatibacter aphrophilus and Bacteroides uniformis. Acta Med Acad. 2015; 44(2):181-5. doi: https:// doi.org/ 10.5644/ama2006-124.

14. Maraki S, Papadakis IS, Chronakis E, Panagopoulos D, Vakis A. Aggregatibacter aphrophilus brain abscess secondary to primary tooth extraction: Case report and literature review. J Microbiol Immunol Infect. 2016; 49(1):119-22. doi: $\quad$ http://dx.doi.org/10.1016/ j.jmii.2013.12.007 